\title{
NetQoPE: A Model-driven Network QoS Provisioning Engine for Distributed Real-time and Embedded Systems*
}

\author{
Jaiganesh Balasubramanian $^{\dagger}$, Sumant Tambe ${ }^{\dagger}$, Balakrishnan Dasarathy ${ }^{\ddagger}$, \\ Shrirang Gadgil ${ }^{\ddagger}$, Frederick Porter ${ }^{\dagger}$, Aniruddha Gokhale ${ }^{\dagger}$, and Douglas C. Schmidt ${ }^{\dagger}$ \\ ${ }^{\dagger}$ Department of EECS, Vanderbilt University, Nashville, TN, USA \\ ¥Telcordia Technologies, Piscataway, NJ, USA
}

\begin{abstract}
This paper provides two contributions to the study of quality of service $(Q o S)$-enabled middleware that supports the network QoS requirements of distributed real-time and embedded (DRE) systems. First, we describe the design and implementation of NetQoPE, which is a model-driven component middleware framework that shields applications from the details of network QoS mechanisms by (1) specifying per-flow network QoS requirements, (2) performing resource allocation and validation decisions (such as admission control), and (3) enforcing per-flow network QoS at runtime. Second, we evaluate the effort required and flexibility of using NetQoPE to provide network QoS assurance to end-to-end application flows. Our results demonstrate that NetQoPE can provide network-level differentiated performance to each application flow without modifying its programming model or source code, thereby providing greater flexibility in leveraging network-layer mechanisms.
\end{abstract}

\section{Introduction}

Emerging trends. Distributed real-time and embedded (DRE) systems, such as shipboard computing systems, supervisory control and data acquisition (SCADA) systems, and enterprise security and hazard sensing subsystems, consist of multiple communication-intensive applications with multiple end-to-end application flows. These systems have network quality of service $(\mathrm{Q} o \mathrm{~S})$ requirements, such as low end-to-end roundtrip latency and jitter, that must be satisfied under varying levels of network connectivity and bandwidth availability. Network QoS mechanisms, such as integrated services (IntServ) [12] and differentiated services (DiffServ) [2], help provide diverse network service levels for applications in DRE systems.

For example, applications can use advanced network QoS mechanisms (e.g., a DiffServ bandwidth broker [3]) to (1) request a network service level and (2) allocate and man-

*This work is supported in part or whole by DARPA Adaptive and Reflective Middleware Systems Program Contract NBCH-C-03-0132. Any opinions, findings, conclusions or recommendations expressed in this material are those of the author(s) and do not necessarily reflect the views of the Department of the Interior, National Business Center, Acquisition Services Division, Southwest Branch or DARPA. age network resources for their remote invocations. Applications invoke remote operations by adding a service levelspecific identifier (e.g., DiffServ codepoint (DSCP)) to the IP packets. DiffServ-enabled network routers parse the IP packets and provide the appropriate service level-specific packet forwarding behavior.

Limitations with current approaches. Although advanced network QoS mechanisms are powerful, it is tedious and error-prone to develop applications that interact directly with low-level network QoS mechanism APIs written imperatively in third-generation languages, such as $\mathrm{C}++$ or Java. To overcome this problem, middleware-based solutions $[22,18,16,4]$ have been developed that allow applications to specify their coordinates (source and destination IP and port addresses) and per-flow network QoS requirements via higher-level frameworks. The middleware frameworks - rather than the applications — are responsible for converting the higher-level QoS specifications into the lower-level network QoS mechanism APIs.

Although middleware frameworks alleviate many accidental complexities of low-level network QoS mechanism APIs, they can still be hard to evolve and extend. In particular, application source code changes may be necessary whenever changes occur to the deployment contexts (source and destination nodes of the applications), per-flow requirements, IP packet identifiers, or the middleware APIs. What is needed, therefore, are middleware-guided network QoS provisioning solutions that (1) are not tied to a particular network QoS mechanism and (2) do not modify application source code to specify and enforce network QoS requirements. These solutions should ideally operate on welldefined system abstractions (e.g., per-flow requirements and source/destination nodes) that do not require programmatically modifying application source code, thereby facilitating application reuse across a wide range of deployment and network QoS contexts.

Solution approach $\rightarrow$ A model-driven component middleware network QoS provisioning framework that uses declarative domain-specific techniques [1] to raise the level of abstraction of DRE system design higher than using imperative third-generation programming languages. A model-driven framework allows system engineers and software developers to perform deployment-time analysis (such as schedulability analysis [10]) of non-functional system 
properties (such as network QoS assurances for end-toend application flows). Such an analysis helps provide deployment-time assurance that application QoS requirements will be satisfied.

This paper describes the Network QoS Provisioning Engine (NetQoPE), which is a model-driven component middleware framework that deploys and configures applications in DRE systems and enforces their network QoS requirements using the four-stage (i.e., design-, pre-deployment, deployment-, and runtime) approach shown in Figure 1. The innovative elements of NetQoPE's four-stage architec-

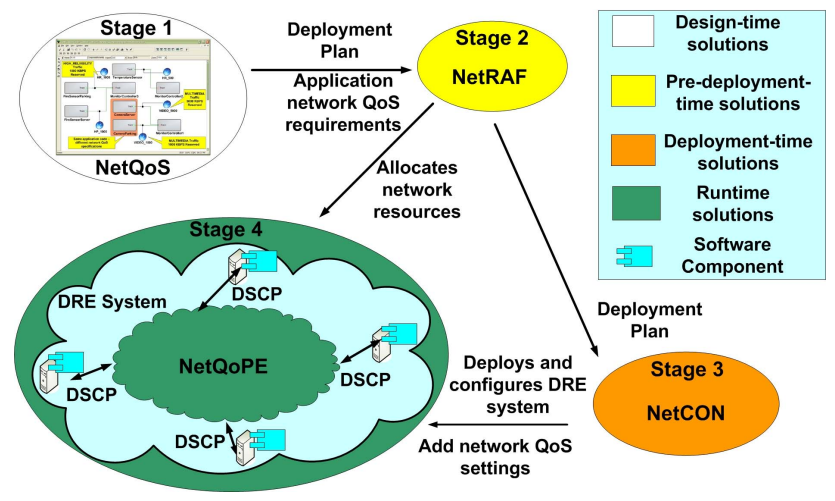

Figure 1: NetQoPE's Four-stage Architecture

ture include the following:

- The Network QoS Specification Language (NetQoS), which is a domain-specific modeling language (DSML) that supports design-time specification of per-flow network QoS requirements, such as bandwidth and delay across a flow. By allowing application developers to focus on functionality-rather than the different deployment contexts (e.g., different bandwidth and delay requirements) where they will be used-NetQoS simplifies the deployment of applications in contexts that have different network QoS needs, e.g., different bandwidth requirements.

- The Network Resource Allocation Framework (NetRAF), which is a middleware-based resource allocator framework that uses the network QoS requirements captured by NetQoS as input at pre-deployment time to help guide QoS provisioning requests on the underlying network QoS mechanism at deployment time. By providing application-transparent, per-flow resource allocation capabilities at pre-deployment-time, NetRAF minimizes runtime overhead and simplifies validation decisions, such as admission control.

- The Network QoS Configurator (NetCON), which is a middleware-based network QoS configurator that provides deployment-time configuration of component middleware containers. NetCON adds flow-specific identifiers (e.g., DSCPs) to IP packets at runtime when applications invoke remote operations. By providing container-mediated and application-transparent capabilities to enforce runtime network QoS, NetCON allows DRE systems to leverage the QoS services of configured routers without modifying application source code.

As shown in the Figure 1, the output of each stage in NetQoPE serves as input for the next stage, which helps automate the deployment and configuration of DRE applications with network QoS support.

Paper organization. The remainder of the paper is organized as follows: Section 2 describes a case study that motivates common requirements associated with provisioning network QoS for DRE systems; Section 3 explains how NetQoPE addresses those requirements via its model-driven component middleware framework; Section 4 evaluates the capabilities provided by NetQoPE; Section 5 compares our work on NetQoPE with related research; and Section 6 presents concluding remarks and lessons learned.

\section{Motivating NetQoPE's Network QoS Pro- visioning Capabilities}

Figure 2 shows a representative DRE system in an office enterprise security and hazard sensing environment, which we use as a case study to demonstrate and evaluate NetQoPE's model-driven, middleware-guided network QoS provisioning capabilities. Enterprises often transport net-

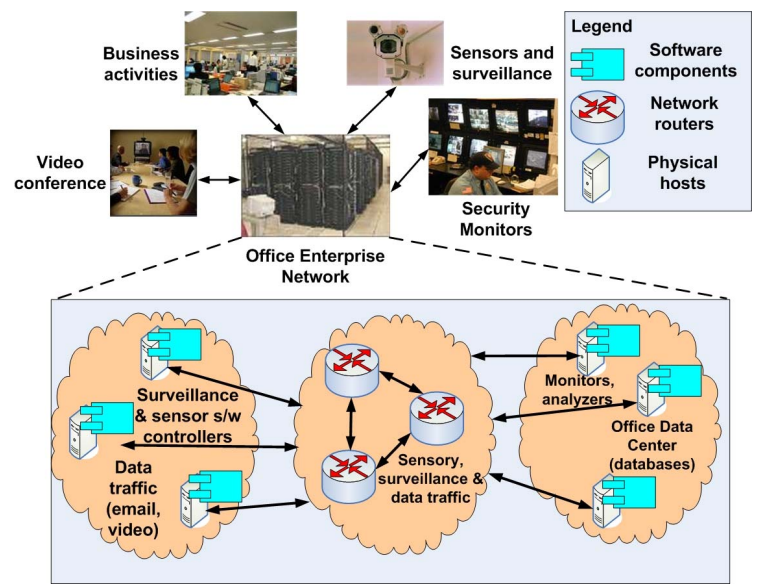

Figure 2: Network Configuration in an Enterprise Security and Hazard Sensing Environment

work traffic using an IP network over high-speed Ethernet. Network traffic in an enterprise can be grouped into several classes, including (1) e-mail, videoconferencing, and normal business traffic, and (2) sensory and imagery traffic of the safety/security hardware (such as fire/smoke sensors) installed on office premises. Our case study makes the common assumption that safety/security traffic is more critical than other traffic, and thus focuses on model-driven, middleware-guided mechanisms to assure the specified QoS for this type of traffic in the presence of other traffic that shares the same network. 
As shown in Figure 2, our case study uses software controllers to manage hardware devices, such as sensors and monitors. Each sensor/camera software controller filters the sensory/imagery information and relays them to the monitor software controllers that display the information. An office enterprise could have lot of sensors and monitors deployed, and each of these communications could have a variety of network QoS requirements. These software controllers were developed using Lightweight CCM (LwCCM) [14] and the traffic between these software controllers uses a bandwidth broker [3] to manage network resources via DiffServ network QoS mechanisms. Although the case study in this paper focuses on DiffServ and LwCCM, NetQoPE is designed for use with other network QoS mechanisms (e.g., IntServ) and component middleware technologies (e.g., J2EE).

Component-based applications in our case study use bandwidth broker services via the following middlewareguided steps: (1) network QoS requirements are specified on each application flow, along with information on the source and destination IP and port addresses, (2) the bandwidth broker is invoked to reserve network resources along the network paths for each application flow, configure the corresponding network routers, and obtain per-flow DSCP values to help enforce network QoS, and (3) remote operations are invoked with appropriate DSCP values added to the IP packets so that configured routers can provide perflow differentiated performance. Section 3 describes the challenges we encountered when implementing these steps in the context of our case study and shows how NetQoPE's four-stage architecture shown in Figure 1 resolves these challenges.

\section{NetQoPE's Multistage Network QoS Provi- sioning Architecture}

As discussed in Section 1, conventional techniques for providing network QoS to applications incur several key limitations, including modifying application source code to (1) specify deployment context-specific network QoS requirements, and (2) integrate functionality from network QoS mechanisms at runtime. This section describes how NetQoPE addresses these limitations via its model-driven, middleware-guided network QoS provisioning architecture.

\subsection{Challenge 1: Alleviating Complexities in QoS Re- quirements Specification}

Context. Each application flow in a DRE system can specify a required level of service (e.g., high priority vs. low priority), the source and destination IP and port addresses, and bandwidth and delay requirements. This information is used to allocate and configure network resources to provide the required QoS.

Problem. Network QoS requirements (such as the band- width and delay requirements mentioned above) can change depending on a deployed context. For example, in our case study from Section 2, multiple fire sensors are deployed at different importance levels and each sensor sends its sensory information to its corresponding monitors. Fire sensors deployed in the parking lot have a lower importance than those in the server room. The sensor-monitor flows thus have different network QoS requirements, even though the reusable software controllers managing the fire sensor and the monitor have the same functionality.

Conventional techniques, such as hard-coded API approaches [4], require application source code modifications for each context. Writing this code manually to specify network QoS requirements is tedious, error-prone, and nonscalable. In particular, it is hard to envision at development time all the contexts in which the source code will be deployed.

\section{Sidebar 1: Overview of Lightweight CORBA Component Model (LwCCM)}

Application functionality in LwCCM is provided through components which collaborate with other components via ports to create component assemblies. Assemblies in LwCCM are described using XML descriptors (mainly the deployment plan descriptor) defined by the OMG D\&C [15] specification. The deployment plan includes details about the components, their implementations, and their connections with other components. The deployment plan also has a placeholder configProperty that is associated with elements (e.g., components, connections) to specify their properties (e.g., priorities) and resource requirements. Components are hosted in containers, which provide the appropriate runtime operating environment (e.g., transactions support) for components to invoke remote operations.

Solution approach $\rightarrow$ Model-driven visual network requirements specification. NetQoPE provides a DSML called the Network QoS Specification Language (NetQoS). DRE system developers can use NetQoS to (1) model component assemblies, (2) assign components to target nodes, and (3) declaratively specify the following deployment context-specific network QoS requirements on the modeled application flows: (a) network QoS classes, such as HIGH PRIORITY (HP), HIGH RELIABILITY (HR), MULTIMEDIA (MM), and BEST EFFORT (BE), (b) bi-directional bandwidth and delay requirements, and (c) selection of transport protocol.

In the context of our case study, NetQoS's network QoS classes correspond to the DiffServ levels of service provided by our Bandwidth Broker [3]. ${ }^{1}$ For example, the HP class

\footnotetext{
${ }^{1}$ NetQoS's DSML capabilities can be extended to provide requirements specification conforming to a different network QoS mechanism, such as IntServ.
} 
represents the highest importance and lowest latency traffic (e.g., fire detection reporting in the server room). The HR class represents traffic with low drop rate (e.g., surveillance data). NetQoS also supports the MM class for sending multimedia data and the BE class for sending traffic with no QoS requirements.

After a model has been created, NetQoS's model interpreter traverses the modeled application structure and generates a deployment plan (described in Sidebar 1). NetQoS's model interpreter also traverses each modeled application flow and augments the deployment plan configProperty tags (also described in Sidebar 1) to express network QoS requirement annotations on the component connections. Section 3.2 describes how NetQoS allocates network resources based on requirements specified in the deployment plan descriptor.

Our case study has certain application flows (e.g., a monitor requesting location coordinates from a fire sensor) where the client needs to control the network priorities at which the requests and replies are sent. If a network QoS provisioning engine provides this capability for applications, real-time actions can be controlled irrespective of network congestion. There are other examples (e.g., a temperature sensor sends temperature sensory information to the monitors), where the servers (in this case the monitors) could control how to receive and act on client requests.

To support these two models, NetQoS can assign the following priority attributes to connections: (1) the CLIENT_PROPAGATED network priority model that allows the clients to dictate the bi-directional priorities, and (2) the SERVER_DECLARED network priority model that allows servers to dictate the bi-directional priorities. NetQoS's model interpreter updates the deployment plan with these priority models for each flow. Section 3.3 explains how NetQoPE's runtime mechanisms honor these priority models when applications invoke remote operations.

Application to the case study. Figure 3 shows a NetQoS model that highlights many of its key capabilities. Multiple

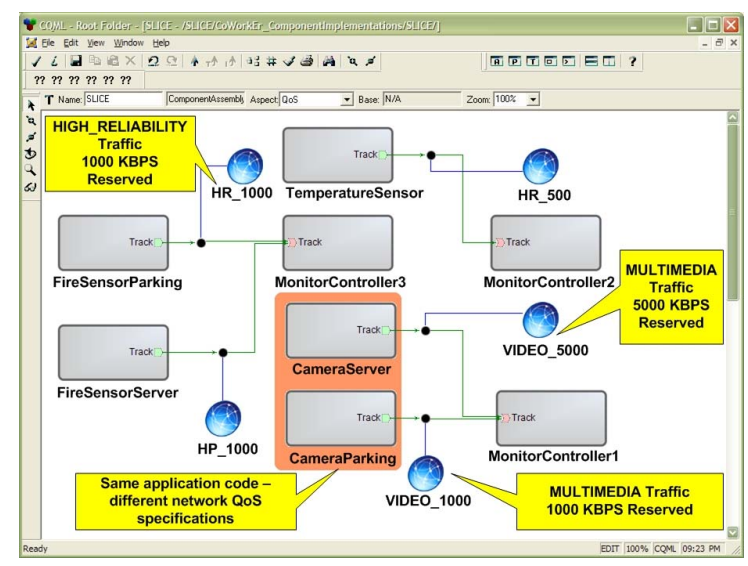

Figure 3: NetQoS Capabilities instances of the same reusable application components (e.g., FireSensorParking and FireSensorServer components) can be annotated with different QoS attributes using an intuitive drag and drop technique. This method of specifying QoS requirements is thus much simpler than modifying application code for each deployment context, as demonstrated in Section 4.2. Moreover, the same QoS attribute (e.g., HR_1000 in Figure 3) can be reused across multiple connections. NetQoS thus increases the scalability of expressing requirements for large numbers of connections that are prevalent in large-scale DRE systems, such as our case study.

\subsection{Challenge 2: Alleviating Complexities in Network Resource Allocation and Configuration}

Context. DRE systems must allocate and configure network resources based on the QoS requirements specified on their application flows so that network QoS assurance can be provided at runtime.

Problem. In our case study, the temperature sensory information from the server room is more important than the information from a conference room. It is undesirable, however, to modify the temperature sensor software controller code to directly interact with a middleware API or network QoS mechanism API since certain deployment contexts (such as the deployment in a conference room) might not require network QoS assurances. Moreover, if application source code is modified to provide resource allocations, decisions on whether to allocate resources or not cannot be determined until the applications are deployed and operational. This approach forces DRE system deployers to stop application components and deploy them on different nodes if required resources cannot be allocated across source and destination nodes.

Solution approach $\rightarrow$ Middleware-based Resource Allocator Framework. NetQoPE's Network Resource Allocator Framework (NetRAF) is a resource allocator engine that allocates network resources for DRE systems using a variety of network QoS mechanisms, such as DiffServ and IntServ. As shown in Figure 4, the NetQoS DSML described in Section 3.1 captures the modeled per-flow network QoS requirements in the form of a deployment plan that is input to NetRAF.

The modeled deployment context could have many instances of the same reusable source code, e.g., the temperature sensor software controller could be instantiated two times: one for the server room and one for the conference room. When using NetQoS, however, application developers annotate only the connection between the instance at the server room and the monitor software controller. Since NetRAF operates on the deployment plan that captures this modeling effort, network QoS mechanisms are used only for the connection on which QoS attributes are added. 


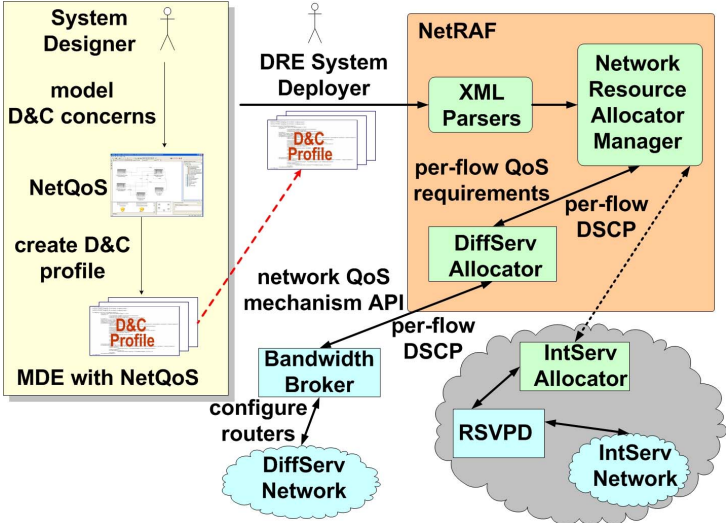

Figure 4: NetRAF's Network Resource Allocation Capabilities

NetRAF thus improves conventional approaches [18] that modify application source code to work with network QoS mechanisms, which can become complex when source code is reused in a wide range of deployment contexts.

NetRAF's Network Resource Allocator Manager accepts application QoS requests at pre-deployment-time and determines the network QoS mechanism (e.g., DiffServ or IntServ) to use to serve the requests. As shown in Figure 4, NetRAF's Network Resource Allocator Manager works with QoS mechanism-specific allocators (e.g., DiffServ Allocator), which shields it from interacting directly with complex APIs for network QoS mechanisms (e.g., DiffServ Bandwidth Broker), thereby enhancing NetQoPE's flexibility.

Multiple allocators (e.g., IntServ Allocator and DiffServ Allocator) can be used by NetRAF's Network Resource Allocator Manager to serve the needs of small-scale deployments (where IntServ and DiffServ are both suitable) and large-scale deployments (where DiffServ often provides better scalability). For example, the shaded cloud connected to the Network Resource Allocator Manager in Figure 4 shows how NetRAF can be extended to work with other network QoS mechanisms, such as IntServ.

Application to the case study. Since our case study is based on DiffServ, NetRAF uses the DiffServ Allocator to allocate network resources. This allocator invokes the Bandwidth Broker's admission control capabilities [3] by feeding it one application flow at a time. If all flows cannot be admitted, NetRAF allows developers an option to modify the deployment context since applications have not yet been deployed. Example modifications include changing component implementations to consume fewer resources or change the source and destination nodes. As demonstrated in Section 4.2, this capability helps NetRAF incur lower overhead than conventional approaches $[22,18]$ that perform validation decisions when applications are deployed and operated at runtime.

NetRAF's DiffServ Allocator instructs the Bandwidth
Broker to reserve bi-directional resources in the specified network QoS classes as described in Section 3.1. The Bandwidth Broker determines the bi-directional DSCPs and NetRAF encodes those values as connection attributes in the deployment plan. In addition, the Bandwidth Broker uses its Flow Provisioner [3] to configure the routers to provide appropriate per-hop behavior when they receive IP packets with the specified DSCP values. Section 3.3 describes how component containers are auto-configured to add these DSCPs when applications invoke remote operations.

\subsection{Challenge 3: Alleviating Complexities in Network QoS Settings Configuration}

Context. After network resources are allocated and network routers are configured, applications in DRE systems need to invoke remote operations using the chosen network QoS settings (e.g., DSCP markings) so that the network layer can differentiate application traffic and provision appropriate QoS to each flow.

Problem. Application developers have historically written code that instructs the middleware to provide the appropriate runtime services, e.g., DSCP markings in IP packets [16]. For example, fire sensors in our case study from Section 2 can be deployed in different QoS contexts that are managed by reusable software controllers. Modifying application code to instruct the middleware to add network QoS settings is tedious, error-prone, and non-scalable because (1) the same application code could be used in different contexts requiring different network QoS settings and (2) application developers might not (and ideally should not) know the different QoS contexts in which the applications are used during the development process. Applicationtransparent mechanisms are therefore needed to configure the middleware to add these network QoS settings depending on the application deployment context.

Solution approach $\rightarrow$ Deployment and runtime component middleware mechanisms. Sidebar 1 describes how LwCCM containers provide a runtime environment for components. NetQoPE's Network QoS Configurator (NetCON) can auto-configure these containers by adding DSCPs to IP packets when applications invoke remote operations. As shown in Figure 5, NetRAF performs network resource allocations, determines the bi-directional DSCP values to use for each application flow and encodes those DSCP values in the deployment plan.

During deployment, NetCON parses the deployment plan and its connection tags to determine (1) source and destination components, (2) the network priority model to use for their communication, (3) the bi-directional DSCP values, and (4) the target nodes on which the components are deployed. NetCON deploys the components on their respective containers and creates the associated object references for use by clients in a remote invocation. When a 


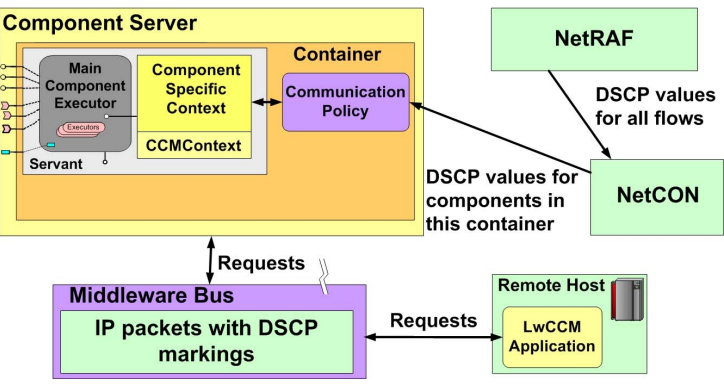

Figure 5: NetCON's Container Auto-configurations

component invokes a remote operation in LwCCM, its container's context information provides the object reference of the destination component. Other component middleware provides similar capabilities via containers, e.g., EJB applications interact with containers to obtain the right runtime operating environment.

NetCON's container programming model can transparently add DSCPs and enforce the network priority models described in Section 3.1. To support the SERVER_DECLARED network priority model, NetCON encodes a SERVER_DECLARED policy and the associated request/reply DSCPs on the server's object reference. When a client invokes a remote operation with this object reference, the client-side middleware checks the policy on the object reference, decodes the request DSCP, and includes it in the request IP packets. In the server-side middleware, before sending the reply, the policy is checked again, and the reply DSCP is added on the IP packets.

To support the CLIENT_PROPAGATED network priority model, NetCON configures the containers to apply a CLIENT_PROPAGATED policy at the point of binding an object reference with the client. In contrast to the SERVER_DECLARED policy, the CLIENT_PROPAGATED policy can be changed at runtime and different clients can access the servers with different network priorities. When the source component invokes a remote operation using the policy-applied object reference, NetCON adds the associated forward and reverse DSCP markings on the IP packets, thereby providing network QoS to the application flow. A container can therefore transparently add both forward and reverse DSCP values when components invoke remote operations using the container services.

Application to the case study. NetCON allows DRE system developers to focus on their application business logic, rather than wrestling with low-level mechanisms for provisioning network QoS. Moreover, NetCON provides these capabilities without modifying application code, thereby simplifying development and avoiding runtime overhead.

\section{Evaluating NetQoPE}

This section evaluates the flexibility of using NetQoPE to provide network QoS assurance to end-to-end application flows and demonstrates how NetQoPE's network QoS provisioning capabilities significantly reduce application development effort incurred by conventional approaches.

\subsection{Evaluation Scenario}

Figure 6 shows key component interactions in the modern office enterprise case study shown in Figure 2 that motivated the design of these evaluations using NetQoPE. Our scenario consists of software components (e.g., a fire sensor controller, monitor controller) developed using the CIAO middleware, which is an open-source LwCCM implementation developed on top of TAO real-time CORBA Object Request Broker (ORB). Figure 6 also shows the underlying network topology with Diffserv enabled routers (e.g., $\mathrm{P}, \mathrm{Q})$, which we configure using an associated Bandwidth Broker [3] software hosted on $C$.

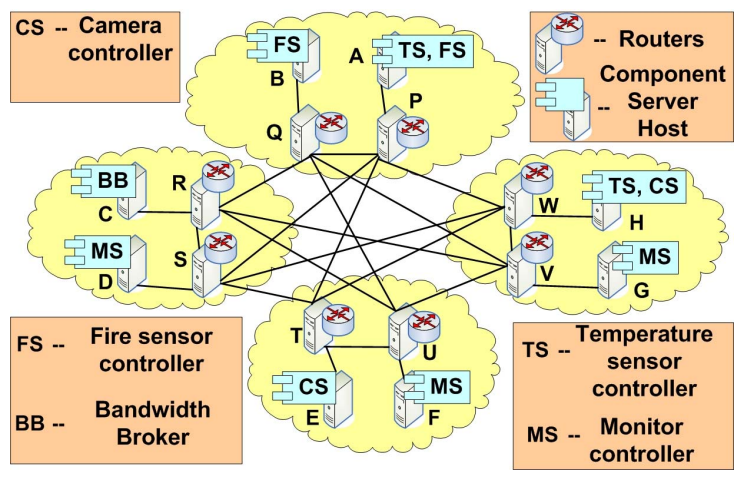

Figure 6: Experimental Setup

In our evaluation scenario, a number of sensory and imagery software controllers sent their monitored information to monitor controllers so that appropriate control actions could be performed by enterprise supervisors monitoring abnormal events. For example, Figure 6 shows two fire sensor controller components deployed on hosts A and B. These components sent their monitored information to monitor controller components deployed on hosts D and F. Communication between these software controllers used one of the traffic classes (e.g., HIGH PRIORITY (HP)) defined in Section 3.1 with the following capacities on all links: $\mathrm{HP}=20 \mathrm{Mbps}, \mathrm{HR}=30 \mathrm{Mbps}$, and $\mathrm{MM}=30 \mathrm{Mbps}$. The BE class used the remaining available bandwidth in the network. The goal of our experiments was to evaluate the flexibility of using NetQoPE to provide network QoS assurance to end-to-end application flows, such as the application flow between the fire sensor controller component on host $\mathrm{A}$ and the monitor controller component on host D.

\subsection{Evaluating NetQoPE's Model-driven QoS Provi- sioning Capabilities}

Rationale. As discussed in Section 3, NetQoPE is designed to provide network QoS to applications in an extensible manner. This experiment evaluates NetQoPE's application- 
transparent network QoS provisioning capabilities.

Methodology. We first identified four flows from Figure 6 whose network QoS requirements are described as follows: (1) a fire sensor controller component on host A uses the high reliability (HR) class to send potential fire alarms in the parking lot to the monitor controller component on host $\mathrm{D}$, (2) a fire sensor controller component on host B uses the high priority (HP) class to send potential fire alarms in the server room to the monitor controller component on host F, (3) a camera controller component on host E uses the multimedia (MM) class and sends imagery information of the break room to the monitor controller component on host $\mathrm{G}$, and (4) a temperature sensor controller component on host $\mathrm{A}$ uses the best effort (BE) class and sends temperature readings to the monitor controller component on host F. The clients dictated the network priority for the requests and replies in all flows except for the temperature sensor and monitor controller component flow, where the server dictated the priority. We used TCP as the transport protocol and $20 \mathrm{Mbps}$ of forward and reverse bandwidth was requested for each type of network QoS traffic.

To compare NetQoPE's methodology of provisioning network QoS for these flows with other existing solutions, we also define a taxonomy for evaluating technologies that provide network QoS assurances to end-to-end DRE application flows. Conventional approaches can be classified as being (1) object-oriented [8, 18, 22, 16], (2) aspectoriented [7], and (3) component middleware-based [4, 19]. We now describe how each approach provides the following functionality needed to leverage network QoS mechanism capabilities:

- Requirements specification. In conventional approaches applications use (1) middleware-based APIs [8, 22], (2) contract definition languages [18, 16], (3) runtime aspects [7], or (4) specialized component middleware container interfaces [4] to specify network QoS requirements. Whenever the deployment context and the associated QoS requirements change, however, application source code must also change, thereby limiting reusability. In contrast, as described in Section 3.1, NetQoS provides domain-specific, declarative techniques that increase reusability across different deployment contexts and alleviate the need to programmatically specify QoS requirements.

- Network resource allocation. Conventional approaches require the deployment of applications before their per-flow network resource requirements can be provisioned by network QoS mechanisms. If the required resources cannot be allocated for those applications they must be stopped, their source code must be modified to specify new resource requirements, and the resource reservation process must be restarted. This approach is tedious since it involves deploying and re-deploying applications multiple times (potentially on different nodes). In contrast, NetRAF handles deployment changes through NetQoS models, as described in Section 3.2. This process occurs during pre-deployment before applications have been deployed, which reduces the effort needed to change deployment topology or application QoS requirements.

- Network QoS enforcement. Conventional approaches modify application source code [16] or programming model [4] to instruct the middleware to enforce runtime QoS for their remote invocations. Applications must therefore be designed to handle two different usecases-to enforce QoS and when no QoS is required-thereby limiting application reusability. In contrast, as described in Section 3.3, NetCON uses a container programming model that transparently enforces runtime QoS for applications without changing their source code or programming model.

Using the conventional approaches and the NetQoPE approach, we now compare the manual effort required to provide network QoS to the 4 end-to-end application flows described above. We decompose the manual effort across the following general steps: (1) implementation, where software developers write code, (2) deployment, where system deployers map (or stop) application components on their target nodes, and (3) modeling tool use, where application developers use NetQoPE to model a DRE application structure and specify per-flow QoS requirements. In our evaluation, a complete QoS provisioning lifecycle consists of specifying requirements, allocating resources, deploying applications, and stopping applications when they are finished.

To compare NetQoPE with manual efforts, we devised a realistic scenario for the 4 end-to-end application flows described above. In this scenario, three sets of experiments were conducted with the following deployment variants:

- In the first variant, all 4 end-to-end application flows were configured with the network QoS requirements as described above.

- In the second variant, to demonstrate the effect of changes in QoS requirements on manual efforts we modified the bandwidth requirements from $20 \mathrm{Mbps}$ to $12 \mathrm{Mbps}$ for each end-to-end flow.

- In the third variant, we demonstrate the effect of changes in QoS requirements and resource (re)reservations taken together on manual efforts. We modified bandwidth requirements of all flows from $12 \mathrm{Mbps}$ to $16 \mathrm{Mbps}$. We also changed temperature sensor controller component to use the high reliability (HR) class instead of the best effort BE class. Finally, we increased the background HR class traffic across the hosts so that the resource reservation request for the flow between temperature sensor and monitor controller components fails. In response, deployment contexts (e.g., bandwidth requirements, source and destination nodes) were changed and resource re-reservation was performed. 
For the first deployment, the manual effort required using conventional approaches involved 10 steps: (1) modify source code for each of the 4 components to specify their QoS requirements (8 implementation steps), (2) deploy all components (1 deployment step), and (3) shutdown all components (1 deployment step). Conversely, the effort required using NetQoPE involved the following 4 steps: (1) model the DRE application structure of all 4 end-to-end application flows using NetQoS (1 modeling step), (2) annotate QoS specifications on each end-to-end application flow (1 modeling step), (3) deploy all components (1 deployment step), and (4) shutdown all components (1 deployment step).

For the second deployment, the effort required using a conventional approach is also 10 steps since source code modifications are needed as the deployment contexts changed (in this case, the bandwidth requirements changed across 4 different deployment contexts). In contrast, the effort required using NetQoPE involves 3 steps: (1) annotate QoS specifications on each end-to-end application flow (1 modeling step), (2) deploy all components (1 deployment step), and (3) shutdown all components (1 deployment step). Application developers also reused NetQoS's application structure model created for the initial deployment, which helped reduce the required efforts by a step.

For the third deployment, the effort required using a conventional approach is 13 steps: (1) modify source code of each of the 8 components to specify their QoS requirements (8 implementation steps), (2) deploy all components (1 deployment step), (3) shutdown the temperature sensor component (1 deployment step - resource allocation failed for the component), (4) modify source code of temperature sensor component back to use BE network QoS class (deployment context change) (1 implementation step), (5) redeploy the temperature sensor component (1 deployment step), and (6) shutdown all components (1 deployment step).

In contrast, the effort required using NetQoPE for the third deployment is 4 steps: (1) annotate QoS specifications on each end-to-end application flow (1 modeling step), (2) begin deployment of all the components, but NetRAF's predeployment-time allocation capabilities determined the resource allocation failure and prompted the NetQoPE application developer to change the QoS requirements (1 predeployment step), (3) re-annotate QoS requirements for the temperature sensor component flow (1 modeling step) (4) deploy all components (1 deployment step), and (5) shutdown all components (1 deployment step).

Table 1 summarizes the step-by-step analysis described above. These results show that conventional approaches incur roughly an order of magnitude more effort than NetQoPE to provide network QoS assurance for end-toend application flows. Closer examination shows that in conventional approaches, application developers spend sub- stantially more effort developing software that can work across different deployment contexts. Moreover, this process must be repeated when deployment contexts and their associated QoS requirements change. Additionally, implementations are complex since the requirements are specified using middleware [22] and/or network QoS mechanism APIs [12].

Further, application (re)deployments are required whenever reservation requests fail. In this experiment, only one flow required re-reservation and that incurred additional effort of 3 steps. If there are large number of flows-and enterprise DRE systems like our case study often have dozens or hundreds of flows - the amount of effort required is significantly more than for conventional approaches.

\begin{tabular}{|c|c|c|c|}
\hline \multirow{2}{*}{ Approaches } & \multicolumn{3}{|c|}{ \# Steps in Experiment Variants } \\
\cline { 2 - 4 } & First & Second & Third \\
\hline \hline NetQoPE & 4 & 3 & 5 \\
\hline Conventional & 10 & 10 & 13 \\
\hline
\end{tabular}

Table 1: Comparison of Manual Efforts Incurred in Conventional and NetQoPE Approaches

In contrast, NetQoPE's “write once, deploy multiple times for different QoS" capabilities increase deployment flexibility and extensibility for environments where many reusable software components are deployed. To provide this flexibility, NetQoS generates XML-based deployment descriptors that capture context-specific QoS requirements of applications. For our experiment, communication between fire sensor and monitor controllers was deployed in multiple deployment contexts, i.e., with bandwidth reservations of $20 \mathrm{Mbps}, 12 \mathrm{Mbps}$, and $16 \mathrm{Mbps}$. In DRE systems like our case study, however, the same communication patterns between components could occur in many deployment contexts.

For example, the same communication patterns could use any of the four network QoS classes (HP, HR, MM, and $\mathrm{BE})$. The communication patterns that use the same network QoS class could make different forward and reverse bandwidth reservations (e.g., 4, 8, or $10 \mathrm{Mbps}$ ). As shown in Table 2, NetQoS auto-generates as much as 1,325 lines of XML code for these scenarios, which would otherwise be handcrafted by application developers.

\begin{tabular}{|c|c|c|c|c|}
\hline \multirow{2}{*}{ Number of communications } & \multicolumn{4}{|c|}{ Deployment contexts } \\
\cline { 2 - 5 } & 2 & 5 & 10 & 20 \\
\hline \hline 1 & 23 & 50 & 95 & 185 \\
\hline 5 & 47 & 110 & 215 & 425 \\
\hline 10 & 77 & 185 & 365 & 725 \\
\hline 20 & 137 & 335 & 665 & 1325 \\
\hline
\end{tabular}

Table 2: Generated Lines of XML Code

These results demonstrate that NetQoPE's network QoS 
provisioning capabilities significantly reduce application development effort incurred by conventional approaches and provides increased flexibility in deploying and provisioning multiple application end-to-end flows under multiple deployment and network QoS contexts.

\section{Related Work}

This section compares our R\&D activities on NetQoPE with related work on middleware-based QoS management and model-based design tools.

Network QoS management in middleware. Prior work on integrating network QoS mechanisms with middleware [22, 18, 16, 8] focused on providing middleware APIs to shield applications from directly interacting with complex network QoS mechanism APIs. Middleware frameworks transparently converted the specified application QoS requirements into lower-level network QoS mechanism APIs and provided network QoS assurances. These approaches, however, modified applications to dictate QoS behavior for the various flows. NetQoPE differs from these approaches by providing application-transparent and automated solutions to leverage network QoS mechanisms, thereby significantly reducing manual design and development effort to obtain network QoS.

QoS management in middleware. Prior research has focused on adding various types of QoS capabilities to middleware. For example, [11] describes J2EE container resource management mechanisms that provide CPU availability assurances to applications. Likewise, 2K [24] provides QoS to applications from varied domains using a component-based runtime middleware. In addition, [4] extends EJB containers to integrate QoS features by providing negotiation interfaces which the application developers need to implement to receive desired QoS support. Synergy [17] describes a distributed stream processing middleware that provides QoS to data streams in real time by efficient reuse of data streams and processing components. These approaches are restricted to CPU QoS assurances or application-level adaptations to resource-constrained scenarios. NetQoPE differs by providing network QoS assurances in a application-agnostic fashion.

Deployment-time resource allocation. Prior work has focused on deploying applications at appropriate nodes so that their QoS requirements can be met. For example, prior work $[13,21]$ has studied and analyzed application communication and access patterns to determine collocated placements of heavily communicating components. Other research $[6,9]$ has focused on intelligent component placement algorithms that maps components to nodes while satisfying their CPU requirements. NetQoPE differs from these approaches by leveraging network QoS mechanisms to allocate network resources at pre-deployment-time and enforcing network QoS at runtime.

Model-based design tools. Prior work has been done on model-based design tools. PICML [1] enables DRE system developers to define component interfaces, their implementations, and assemblies, facilitating deployment of LwCCM-based applications. VEST [20] and AIRES [10] analyze domain-specific models of embedded real-time systems to perform schedulability analysis and provides automated allocation of components to processors. SysWeaver [5] supports design-time timing behavior verification of real-time systems and automatic code generation and weaving for multiple target platforms. In contrast, NetQoPE provides model-driven capabilities to specify network QoS requirements on DRE system application flows, and subsequently allocate network resources automatically using network QoS mechanisms. NetQoPE thus helps assure that application network QoS requirements are met at deployment-time, rather than design-time or runtime.

\section{Concluding Remarks}

This paper describes the design and evaluation of NetQoPE, which is a model-driven component middleware framework that manages network QoS for applications in DRE systems. The lessons we learned developing NetQoPE and applying it to a representative DRE system case study thus far include:

- NetQoPE's domain-specific modeling languages help capture per-deployment network QoS requirements of applications so that network resources can be allocated appropriately. Application business logic consequently need not be modified to specify deployment-specific QoS requirements, thereby increasing software reuse and flexibility across a range of deployment contexts.

- Programming network QoS mechanisms directly in application code requires the deployment and running of applications before they can determine if the required network resources are available to meet QoS needs. Conversely, providing these capabilities via NetQoPE's modeldriven, middleware framework helps guide resource allocation strategies before application deployment, thereby simplifying validation and adaptation decisions.

- NetQoPE's model-driven deployment and configuration tools help transparently configure the underlying component middleware on behalf of applications to add contextspecific network QoS settings. These settings can be enforced by NetQoPE's runtime middleware framework without modifying the programming model used by applications. Applications therefore need not change how they communicate at runtime since network QoS settings can be added transparently.

- NetQoPE's strategy of allocating network resources to applications before they are deployed may be too limiting for certain types of DRE systems. In particular, applications in open DRE systems [23] might not consume their resource allotment at runtime, which may underutilize system 
resources. We are therefore extending NetQoPE to overprovision resources for applications on the assumption that not all applications will use their allotment. If runtime resource contentions occur, we are also developing dynamic resource management strategies that can provide predictable network performance for mission-critical applications.

NetQoPE's model-driven middleware platforms and tools, except the Bandwidth Broker used in the experiments, are available in open-source format from www. dre. vanderbilt.edu/cosmic, and along with the CIAO component middleware available at www.dre.vanderbilt. edu.

\section{References}

[1] K. Balasubramanian, J. Balasubramanian, J. Parsons, A. Gokhale, and D. C. Schmidt. A Platform-Independent Component Modeling Language for Distributed Real-time and Embedded Systems. Journal of Computer Systems Science, 73(2):171-185, 2007.

[2] S. Blake, D. Black, M. Carlson, E. Davies, Z. Wang, and W. Weiss. An Architecture for Differentiated Services. Internet Society, Network Working Group RFC 2475, pages 1-36, Dec. 1998.

[3] B. Dasarathy, S. Gadgil, R. Vaidhyanathan, K. Parmeswaran, B. Coan, M. Conarty, and V. Bhanot. Network QoS Assurance in a Multi-Layer Adaptive Resource Management Scheme for Mission-Critical Applications using the CORBA Middleware Framework. In RTAS 2005, San Francisco, CA, Mar. 2005. IEEE.

[4] M. A. de Miguel. Integration of QoS Facilities into Component Container Architectures. In Proceedings of the Fifth IEEE International Symposium on Object-Oriented RealTime Distributed Computing (ISORC 2002), 2002.

[5] D. de Niz, G. Bhatia, and R. Rajkumar. Model-Based Development of Embedded Systems: The SysWeaver Approach. In Proc. of RTAS'06, pages 231-242, Washington, DC, USA, August 2006.

[6] D. de Niz and R. Rajkumar. Partitioning Bin-Packing Algorithms for Distributed Real-time Systems. International Journal of Embedded Systems, 2005.

[7] G. Duzan, J. Loyall, R. Schantz, R. Shapiro, and J. Zinky. Building Adaptive Distributed Applications with Middleware and Aspects. In Proc. of AOSD '04, pages 66-73, New York, NY, USA, 2004.

[8] M. A. El-Gendy, A. Bose, S.-T. Park, and K. G. Shin. Paving the First Mile for QoS-dependent Applications and Appliances. In Proc. of IWQOS'04, Montreal, Canada, June 2004.

[9] S. Gopalakrishnan and M. Caccamo. Task Partitioning with Replication upon Heterogeneous Multiprocessor Systems. In RTAS '06, pages 199-207, San Jose, CA, USA, 2006.

[10] Z. Gu, S. Kodase, S. Wang, and K. G. Shin. A ModelBased Approach to System-Level Dependency and Realtime Analysis of Embedded Software. In RTAS'03, pages 78-85, Washington, DC, May 2003. IEEE.

[11] M. Jordan, G. Czajkowski, K. Kouklinski, and G. Skinner. Extending a J2EE Server with Dynamic and Flexible Resource Management. In Proceedings of the
ACM/IFIP/USENIX International Middleware Conference, Toronto, Canada, 2004.

[12] L. Zhang and S. Berson and S. Herzog and S. Jamin. Resource ReSerVation Protocol (RSVP) Version 1 Functional Specification. Network Working Group RFC 2205, pages 1-112, Sept. 1997.

[13] D. Llambiri, A. Totok, and V. Karamcheti. Efficiently Distributing Component-Based Applications Across Wide-Area Environments. In Proc. of ICDCS'03, 2003.

[14] Object Management Group. Light Weight CORBA Component Model Revised Submission, OMG Document realtime/03-05-05 edition, May 2003.

[15] OMG. Deployment and Configuration of Component-based Distributed Applications, v4.0, Document formal/2006-0402 edition, Apr. 2006.

[16] R. Schantz and J. Loyall and D. Schmidt and C. Rodrigues and Y. Krishnamurthy and I. Pyarali. Flexible and Adaptive QoS Control for Distributed Real-time and Embedded Middleware. In Proc. of Middleware'03, Rio de Janeiro, Brazil, June 2003. IFIP/ACM/USENIX.

[17] T. Repantis, X. Gu, and V. Kalogeraki. Synergy: SharingAware Component Composition for Distributed Stream Processing Systems. In Proc. of Middleware 2006.

[18] R. Schantz, J. Zinky, D. Karr, D. Bakken, J. Megquier, and J. Loyall. An Object-level Gateway Supporting IntegratedProperty Quality of Service. ISORC, 00:223, 1999.

[19] P. Sharma, J. Loyall, G. Heineman, R. Schantz, R. Shapiro, and G. Duzan. Component-Based Dynamic QoS Adaptations in Distributed Real-time and Embedded Systems. In Proceedings of the International Symposium on Distributed Objects and Applications (DOA), Agia Napa, Cyprus, Oct. 2004.

[20] J. A. Stankovic, R. Zhu, R. Poornalingam, C. Lu, Z. Yu, M. Humphrey, and B. Ellis. Vest: An aspect-based composition tool for real-time systems. In Proc. of RTAS'03, page 58, Washington, DC, USA, 2003.

[21] C. Stewart and K. Shen. Performance Modeling and System Management for Multi-component Online Services. In Proc. of NSDI'05, Boston, MA, pages 71-84, May 2005.

[22] P. Wang, Y. Yemini, D. Florissi, and J. Zinky. A Distributed Resource Controller for QoS Applications. In Proceedings of the Network Operations and Management Symposium (NOMS 2000). IEEE/IFIP, Apr. 2000.

[23] X. Wang, D. Jia, C. Lu, and X. Koutsoukos. DEUCON: Decentralized End-to-End Utilization Control for Distributed Real-Time Systems. Parallel and Distributed Systems, IEEE Transactions on, 18(7):996-1009, 2007.

[24] D. Wichadakul, K. Nahrstedt, X. Gu, and D. Xu. 2K: An Integrated Approach of QoS Compilation and Reconfigurable, Component-Based Run-Time Middleware for the Unified QoS Management Framework. In Proc. of Middleware'01, 2001. 